\title{
Natural Bioproducts and Their Potential Preservative Properties in Food Industry ${ }^{+}$
}

\author{
Paul Begea 1, Nicoleta Radu 1,2,*, Mariana Constantin 2, Gelu Vasilescu 2, Iulia Raut 2, \\ Ovidiu Popa ${ }^{1}$, Marilena Gabriela Zaharie ${ }^{3}$, Lucia Parvu ${ }^{4}$ and Mihaela Begea ${ }^{5}$ \\ 1 Biotechnology Faculty, University of Agronomic Sciences and Veterinary Medicine of Bucharest, \\ 59 Bd. Mărăști, 050097 Bucharest, Romania; elabegea@gmail.com (P.B.); ovid_popa@yahoo.com (O.P.) \\ 2 National Institute for Chemistry and Petrochemistry R\&D of Bucharest, Biotechnology Department, \\ 202 Spl. Independentei, 060021 Bucharest, Romania; marriconstantin@yahoo.com (M.C.); \\ gelu_gvp@yahoo.com (G.V.); iulia_rt@yahoo.com (I.R.) \\ 3 National Institute for Medico-Military R\&D “Cantacuzino”, Diagnosis Reagents and Culture Media, \\ Department, 103 Spl. Independentei, 500240 Bucharest, Romania; mzaharie@yahoo.com \\ 4 National Institute for Chemical Pharmaceutical R\&D of Bucharest, Biotechnology Department, \\ 112 CAlea Vitan, 031299 Bucharest, Romania; lucia.pirvu@yahoo.com \\ 5 Biotechnical System Engineering Department, University Politehnica of Bucharest, 313 Splaiul \\ Independentei, 060042 Bucharest, Romania; ela_begea@yahoo.com \\ * Correspondence: nicolbiotec@yahoo.com; Tel.: +40-730728694 \\ + Presented at the 15th International Symposium "Priorities of Chemistry for a Sustainable Development" \\ PRIOCHEM, Bucharest, Romania, 30th October-1st November 2019.
}

Published: 16 October 2019

Keywords: Plantaginaceae; preservative properties; citrus fruits

Phytosanitary products obtained from natural extracts represent an alternative, especially in the case of preservation of the products obtained from organic farming. The purpose of this work was to test the antifungal activity of three extracts obtained from a plant from Plantaginaceae family, obtained according to the methodology presented by Radu et al. [1]. The tests were performed in vitro on phytopathogenic fungi that cause alteration of the citrus fruits, such as Penicillium sp.; and Aspergillus $s p$. The methodology used was that of diffusive discs impregnated in sterile solutions containing $3 \%$ of the solid extract, dissolved into an inert solvent (respectively Dimethyl Sulfoxide) [2-4]. After inoculation with the tested microorganism and treatment with the analyzed phytoextract, the growth of microorganisms was monitored for two weeks. The obtained results showed that the tested extracts inhibit the development of the species of Penicillium citrinum, Penicillium digitatum, and Aspergillus niger. These effects have maintained for $72 \mathrm{~h}$ in the case of the above microorganisms. After 2 weeks the antifungal effect of the analyzed extracts was reduced at $78.5 \%$ in the case of Penicillium citrinum, at $83.5 \%$ in the case of Penicillium digitatum and respectively at $57.9 \%$ in the case of Aspergillus niger. In conclusion, the best results are obtained at the treatment of Penicillium species which attack the citrus fruits, with studied plant extracts. Here is needed a deeper research, in order to develop a bioproducts from Plantaginaceae indigenous plants, with a role in protecting and preserving of citrus fruits.

Acknowledgments: This paper was prepared with the financial support of the project PFE no 31/2018.

\section{References}

1. Balouiri, M.; Sadiki, M.; Ibnsouda, S.K. Methods for in vitro evaluating antimicrobial activity: A review. J. Pharm. Anal. 2016, 6, 71-79. 
2. Radu, N.; Ghita, I; Rau, I. Therapeutic Effect of Polysaccharides from Plantago Species. Mol. Cryst. Liq. Cryst. 2010, 523, 236-246.

3. Radu, N.; Voicescu, M.; Radu, E.; Tanasescu, C. Biomaterial with antioxidant and antifungal activities, obtained from romanian indigenous plants. Mol. Cryst. Liq. Cryst. 2017, 655, 243-249.

4. Radu, N.; Mariana Voicescu, M.; Radu, E.; Tanasescu, C. Zingiber officinale based bioproduct. Properties and influence on some cellulolytic and keratinolytic fungi. Mol. Cryst. Liq. Cryst. 2017, 655, 103-113.

(C) 2019 by the authors. Licensee MDPI, Basel, Switzerland. This article is an open access article distributed under the terms and conditions of the Creative Commons Attribution (CC BY) license (http://creativecommons.org/licenses/by/4.0/). 